\title{
An edge fast-ion D-alpha system installed at ASDEX Upgrade
}

\author{
A. Jansen van Vuuren, ${ }^{1, \text { a) }}$ B. Geiger, ${ }^{2}$ A.S. Jacobsen, ${ }^{3}$ M. Cavedon, ${ }^{1}$ R. Dux, ${ }^{1}$ H. Köhnlein, ${ }^{1}$ and the ASDEX \\ Upgrade Team ${ }^{1, b)}$ \\ 1) Max-Plank-Institut für Plasmaphysik, D-85748 Garching, Germany \\ 2) University of Wisconsin, WI-53706 Madison, USA \\ ${ }^{3)}$ Culham Science Centre, OX14 3DB Abingdon, UK
}

(Dated: 13 September 2019)

An edge fast-ion D-alpha (FIDA) system consisting of lines of sight optimised for edge measurements and a newly designed prototype spectrometer has been installed at ASDEX Upgrade. The system allows for measurements of the full deuterium Balmer alpha spectrum with either good radial coverage or exposure times below $200 \mu \mathrm{s}$. It uses a bar, placed in front of the EM-CCD camera, to partially block the intense unshifted D-alpha light which permits simultaneous measurements of the FIDA and unshifted D-alpha emission. Thus not only active FIDA radiation, present during neutral beam injection (NBI) can be studied, but also detailed investigations of passive FIDA light are possible, since the unshifted D-alpha emission contains information on the density of background neutrals. First measurements show significant levels of active and passive FIDA radiation in discharges with large edge fast-ion populations from off-axis NBI heating. In addition, a particularly strong response of the passive emission to edge localised modes is observed, well explained by modifications of the edge-neutral density as well as the fast-ion density.

\section{INTRODUCTION}

Good confinement of fast ions is important for fusion devices to ensure efficient plasma heating and current drive. In particular, good confinement of fast-ions in the outer region of the plasma is important since this region has the largest volume and thus, contains a significant amount of fast particles that could, if lost not only degrade plasma performance but also damage first wall components in case of localised losses ${ }^{1}$. However, fast-ion diagnostics in fusion experiments are typically optimised for the plasma core. Such diagnostics include fast-ion loss detectors (FILD), collective Thomson scattering, neutron emission spectroscopy, neutral particle analysers and a fast-ion D-alpha spectroscopy (FIDA) ${ }^{2}$. Except for FILD these diagnostics are optimised for core fast-ion measurements and have limited sensitivity to the confined fast-ion content in the edge region. However, FIDA spectroscopy has the potential to be extended to the edge region without extensive effort.

FIDA can be characterised as charge exchange recombination spectroscopy (CXRS) applied to investigate the distribution function of confined fast ions. The technique relies on measuring the Doppler shifted Balmer alpha emission from charge exchange $(\mathrm{CX})$ reactions between fast hydrogenic ions and donor neutrals ${ }^{3}$, with systems installed on multiple fusion devices ${ }^{4-10}$. The large Doppler shift of the radiation of neutralised energetic particles separates their emission from other Balmer-alpha contributions such as the beam and halo emission and makes the measurement of the typically weak fast-ion emission possible. The measured spectral shape contains information on the fast-ion distribution in pitch and energy and the intensity provides information on the density of fast ions and neutrals. Here pitch is defined as the velocity component of the fast ions parallel to the magnetic field over

\footnotetext{
a)Electronic mail: ajvv@ipp.mpg.de

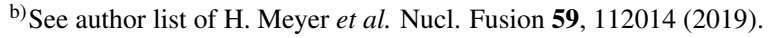

the speed of the fast ions, and it is defined positive in the cocurrent direction. A quantitative interpretation of the measured spectra requires forward modelling ${ }^{11}$ or tomographic reconstructions ${ }^{12}$.

Localised active FIDA measurements can be obtained through the use of an NBI beam to provide a source of donor neutrals. Additionally line integrated passive FIDA due to CX of fast ions with background neutrals is present, especially along the edge where the density of background neutrals is highest.

On the DIII-D tokamak edge measurements have been reported by Zeeland et al. ${ }^{13}$ while passive FIDA has been used as a tool to investigate both the fast-ion and edge neutral densities on MAST ${ }^{7,14}$, DIII-D ${ }^{15}$ and NSTX ${ }^{16}$. Significant passive FIDA during off-axis heating on ASDEX Upgrade has been reported by Geiger et al. ${ }^{4}$ however on core lines of sight (LOS).

Here, the installation of an edge-FIDA system at ASDEX Upgrade is discussed. First the lines of sight geometry will be described, followed by a detailed introduction to the spectrometer design. The calibration procedures will be discussed and finally first measurements during experiments with edge localised modes (ELM) are presented.

\section{LINES OF SIGHT}

The availability of in-vessel optics from an existing edge CXRS system ${ }^{17,18}$ (see figure 1) has opened the possibility to extend existing FIDA measurements to the plasma edge. The optics define 22 toroidal lines of sight that cross a $60 \mathrm{kV}$, $2.5 \mathrm{MW}$ heating beam (Q3) in the steep plasma gradient region. The lines of sight are tangential to the flux surfaces and thereby offer high spatial resolution. The radial resolution of FIDA measurements has been calculated using the FIDASIM Monte Carlo code ${ }^{11,19}$, which includes that neutralised fast ions might travel a considerable distance before emitting their characteristic D-alpha radiation. In FIDASIM, kinetic profiles and a TRANSP ${ }^{20}$ - predicted fast-ion distribution func- 

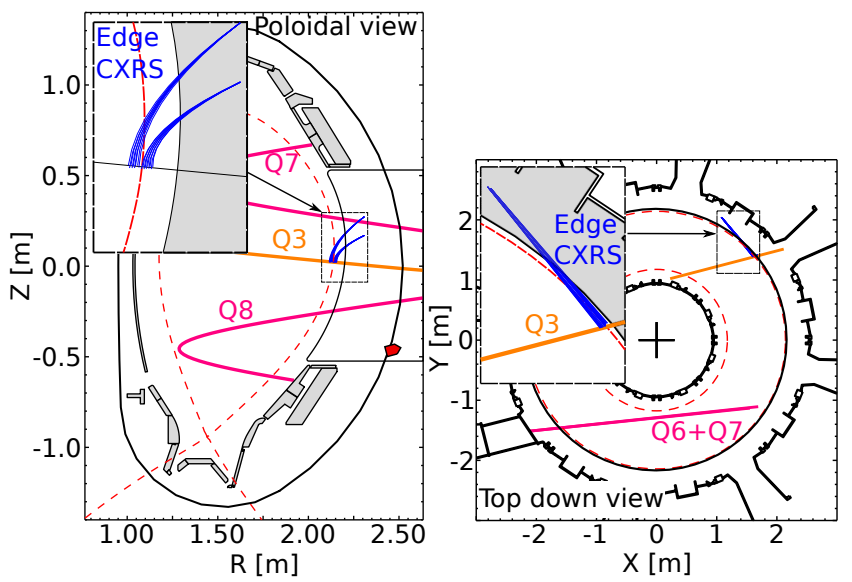

FIG. 1: Poloidal and top down view of the edge CXRS lines of sight used to expand the existing ASDEX Upgrade FIDA system.

tion have been considered from a representative experiment, discussed in section VI. An exponential decay of the fast-ion density with a radial decay length of $2 \mathrm{~cm}$ is assumed outside the separatrix. Figure 2 shows the positions in the poloidal plane where simulated fast ions have become neutralised, before travelling into the view of a given line of sight and contributing to the radiation collected. Here, the simulation data for the innermost and outermost LOS are given. The combined NBI beam and halo neutral density is indicated by black contour lines and the separatrix is provided in red. As can be seen, the region from where information on fast-ions is obtained is localised along the LOS with an extent of about $3 \mathrm{~cm}$ in the radial direction. Figure 3 shows the positions binned to the normalised poloidal flux coordinate, $\rho$, and fitted with a Gaussian function. The average FWHM of the emission is 0.03 in terms of the normalised poloidal flux or $1.5 \mathrm{~cm}$ when mapped in radius.

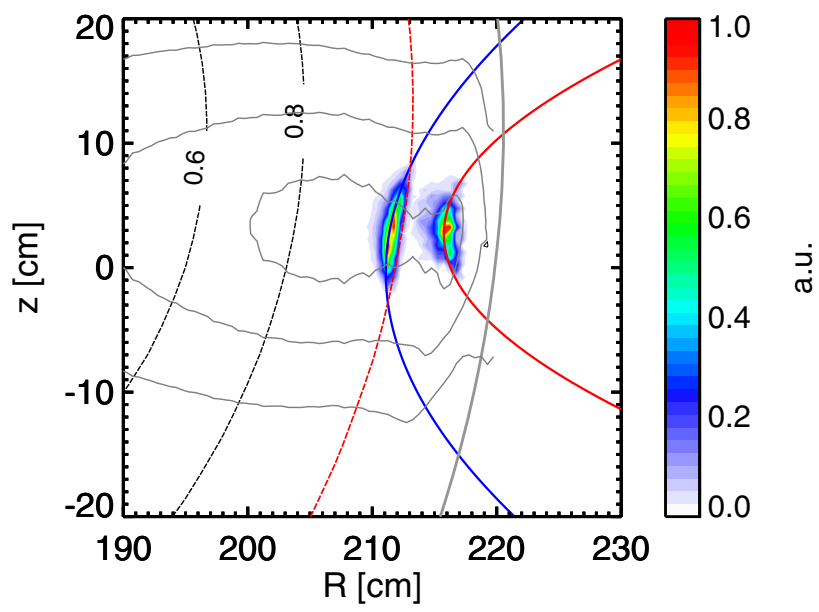

FIG. 2: Poloidal view of active FIDA emission observed by two edge LOS calculated by FIDASIM.

The fast-ion velocity-space sensitivity of the edge-FIDA

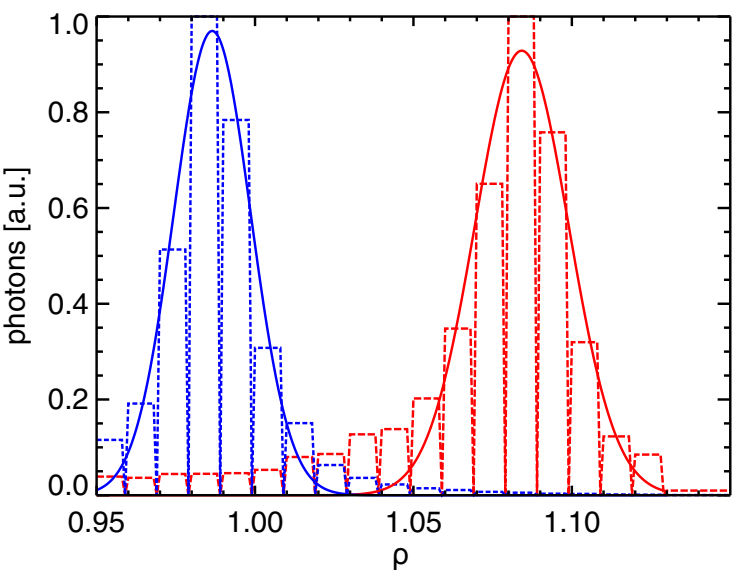

FIG. 3: Active FIDA location calculated by FIDASIM mapped and binned with respect to the normalised poloidal flux coordinate. A Gaussian fit is used to describe the radial width.

system is illustrated in figure 4 in terms of weight functions. The weight functions have been calculated by FIDASIM and display the possible locations in velocity space of the fast ions which can emit detectable light for a given line of sight and wavelength range. As can be seen, the toroidal LOS are largely sensitive to co- or counter-rotating fast ions, for wavelengths with Doppler shifts well separated from the un-shifted Balmer alpha radiation of $\mathrm{D}$ at $656.1 \mathrm{~nm}$.

\section{NEW EDGE FIDA SPECTROMETER DESIGN}

A new and dedicated spectrometer has been developed for edge-FIDA measurements being able to observe red and blue shifted radiation simultaneously. In addition, the primary design criteria of the spectrometer was to be able to measure and resolve the spectral shape and intensity of the FIDA and the unshifted D-alpha emission.

Measuring the unshifted D-alpha emission is important as it provides valuable information on the background neutral density along a LOS. In particular when studying passive FIDA light this becomes essential. However, simultaneously measuring both, the FIDA and unshifted D-alpha contributions is challenging since their intensities can differ by several orders of magnitude (see section VI). Thus, a spectrometer with high dynamic range is required.

Moreover, high temporal $(<1 \mathrm{kHz})$ and moderate spectral resolution $(<0.2 \mathrm{~nm})$ are required. While high temporal resolution is necessary to be able to resolve edge instabilities, the spectral resolution is necessary to identify possible impurity line emissions in the spectrum and to exclude them from the analysis.

The new prototype spectrometer follows a high photon throughput Czerny-Turner like design using lenses instead of mirrors. A gold coated plane holographic grating with 1760 lines/mm mounted on a rotational stage (Newport 


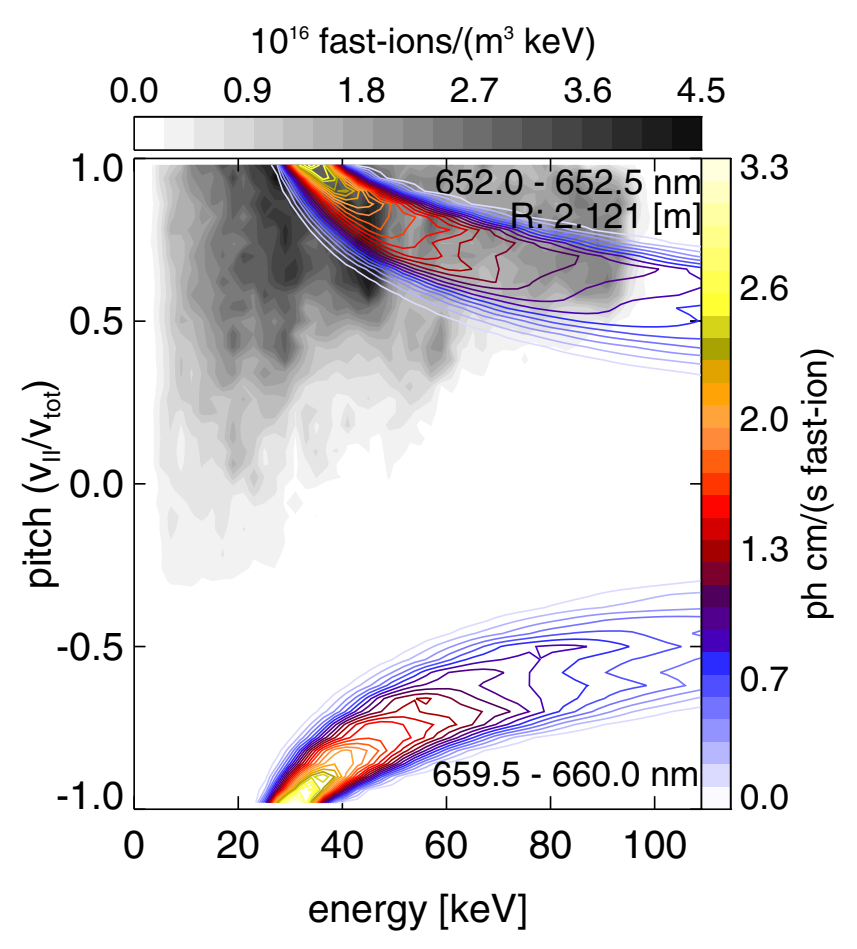

FIG. 4: FIDA weight functions (in colour) calculated by FIDASIM for an edge toroidal LOS. The wavelength range is well separated from the beam emission. The grey contours illustrate a fast-ion distribution as calculated by TRANSP for an off axis NBI heated discharge discussed in section VI.

RVS80CC ${ }^{21}$ ) is used to allow fine adjustments or modifications of the central wavelength. Moreover, two Sigma EX $\mathrm{f} / 2.8$ objective lenses with focal lengths of $300 \mathrm{~mm}$ are used. The spectrometer opening angle $(\phi)$ is $25^{\circ}$ as illustrated in figure 5. The figure illustrates the working principle of the design whereby light from a bundle of $400 \mu \mathrm{m}$ fibres, stacked vertically, enters the spectrometer through a slit. The light reaching the first lens is collimated and directed onto the grating. The grating disperses the light according to wavelength in the horizontal plane. The light reaching the second objective lens is focused onto a (Princeton Instruments ProEM ${ }^{22}$ ) camera with an electron multiplying charge-coupled device (EMCCD) chip. The light sensitive area of the EM-CCD chip is $1024 \times 1024$ pixels with a pixel size of $13 \mu \mathrm{m}$. The spectral width of the spectrometer measuring at $656 \mathrm{~nm}$ is approximately $17 \mathrm{~nm}$ with an average dispersion of $0.017 \mathrm{~nm} /$ pixel.

The high quantum efficiency of the EM-CCD chip is ideal for low light acquisition with a dynamic range of 16 bits. To reduce thermal noise (dark charge), the chip is cooled to -55 ${ }^{\circ} \mathrm{C}$ with a built in Peltier cooler. On chip multiplication (gain of 20 typically selected) of the photo generated charge further reduces the readout noise. This however reduces the effective dynamic range, which requires that the bright, unshifted D-alpha light be strongly dimmed. Therefore a $2 \mathrm{~mm}$ wide 3D printed blocking-bar is positioned in front of the CCD chip, as shown in figure 6 . The purpose of the bar is to dim but not completely block the incident light within a narrow

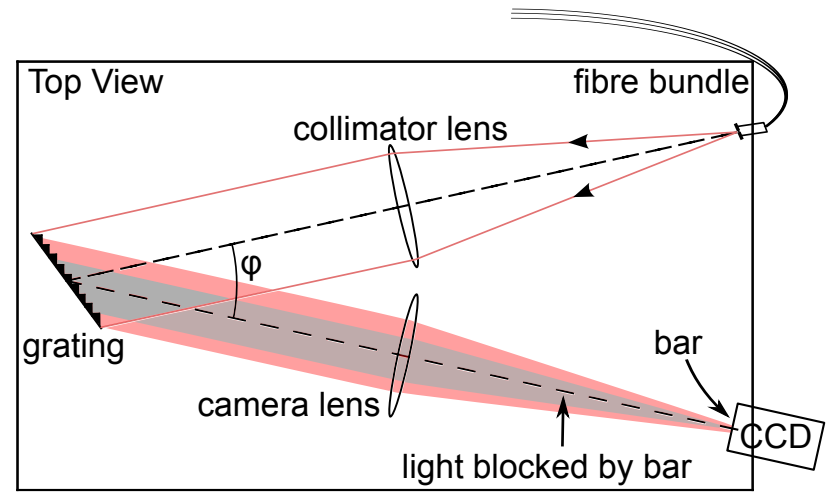

FIG. 5: Edge FIDA spectrometer conceptual design.

wavelength range. The bar is placed directly in contact with the protective glass of the camera, $8 \mathrm{~mm}$ in front of the chip. The triangular cross-section of the bar provides a well defined blocking plane at the back of the bar. The bar affects the spectrum in a $6 \mathrm{~nm}$ wide range as can be seen from the effective transmission curve in figure 7 . The blocking width can be increased by moving the bar further from the chip, however this also increases the transmission of the affected region. The spectral shape of the measured spectra can be recovered by applying the inverse of the transmission curve when applying the intensity calibration. The use of blocking wires using an intermediate image is commonly applied in FIDA spectroscopy systems ${ }^{5,23,24}$, however the approach used here has the advantage that a second correcting lens is not necessary, which allows for better light throughput and better imaging quality.
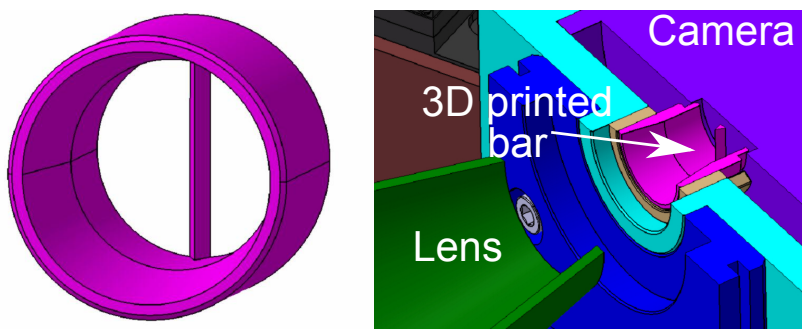

FIG. 6: (Left) CAD drawing of the 3D printed bar designed to obstruct light within a narrow wavelength band. (Right)

The bar is positioned directly in front of the CCD chip.

\section{CAMERA READOUT MODES}

Two different readout options of the camera are used here. First, the frame-transfer mode, typically applied by the suite of CXRS systems ${ }^{25}$ at ASDEX Upgrade allows for continuous imaging. The EM-CCD chip has an additional region of $1024 \times 1037$ pixels that are covered and not illuminated, as illustrated in figure 8. After an exposure the photo-electrons (charge) are shifted row by row to this masked region, at a rate of $0.7 \mu$ s per row. Once all of the charges have been shifted 


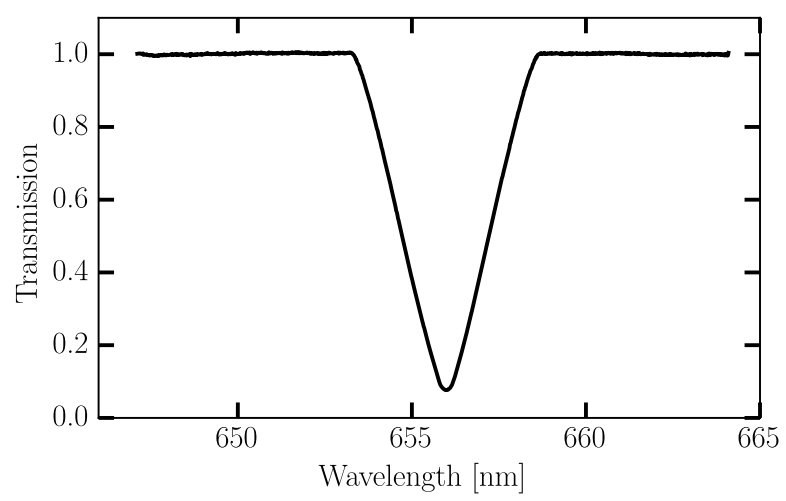

FIG. 7: Measured transmission curve due to the blocking bar. The transmission in the central part is strongly reduced by the $2 \mathrm{~mm}$ thick bar, placed in front of the CCD chip.

to this area the following exposure starts. During this time the charge in the masked region is read out by the serial registers and converted to digital signals at a rate of $20 \mathrm{MHz}$. To reduce the read out time regions of interest (ROI) covering each imaged fibre are defined serving as the spectrometer channels. During read out the charge of each channel is binned in the vertical direction allowing for faster operation. The binning of charge also increases the signal to noise ratio (SNR). The size of the camera chip can accommodate up to 21 fibres with the lowest readout time and thus exposure time of $3.1 \mathrm{~ms}$. The readout time is reduced to $2.5 \mathrm{~ms}$ when 10 fibres are used.

Second, the spectra-kinetics mode can be used, which allows for significantly faster exposure times (down to the $\mu \mathrm{s}$ range) at the cost of having only a single channel available adjacent to the non-illuminated part of the chip. Spectra-kinetics takes advantage of the fast transfer and binning of charge to the covered part of the chip. After an exposure the charge collected in the ROI is binned and shifted to this region where it is stored in one of the 1037 rows. Thus, 1037 exposures can be performed continuously without the slow readout of the data. The frame rate is determined by the combined vertical shift $\left(t_{\text {shift }}\right)$ and programmed exposure $\left(t_{\text {exp }}\right)$ time. The shift time is determined by the size of the ROI which for 120 rows would be $84 \mu \mathrm{s}$. Here, we use an exposure time of $200 \mu \mathrm{s}$, translating into a time-window of about $\left(\left(t_{\exp }+t_{\text {shift }}\right) \times 1037 \approx\right) 300$ $\mathrm{ms}$, since the lowest useful exposure time is dictated by the achievable SNR. The low photon integrated flux during the fast acquisitions increases the significance of readout noise. It should be noted that this noise source can be greatly reduced by reading out not with $20 \mathrm{MHz}$ but with only $10 \mathrm{MHz}$, at the cost of a longer dead-time between bursts but with no effect on time resolution within a burst. Furthermore it is important that only the defined ROI is illuminated during the measurement, since the charge collected in the pixels below the ROI cannot be removed between exposures. Fibres imaged to regions not covered by the ROI are therefore unplugged and covered when the system is operated in the spectra-kinetics mode.
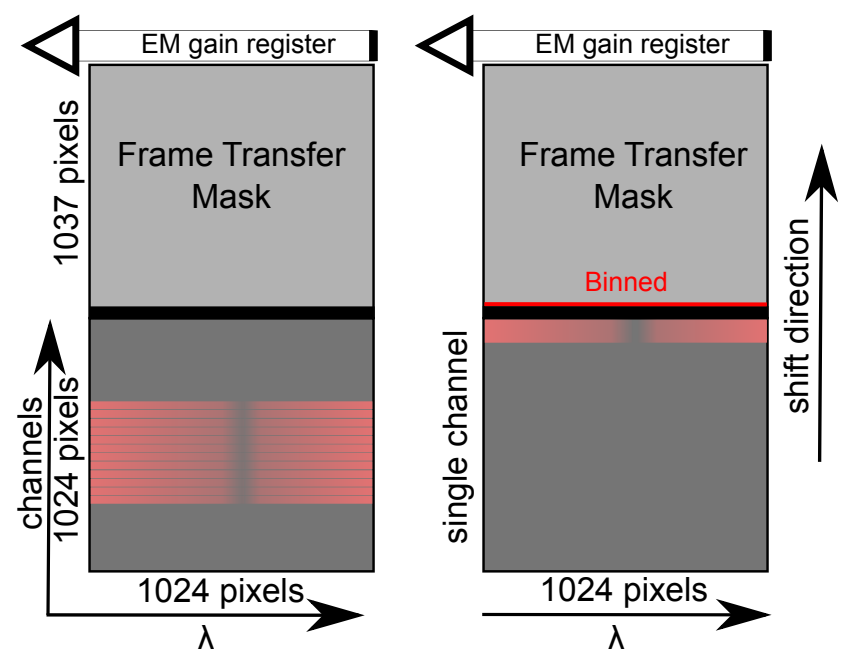

FIG. 8: Diagram displaying the difference in configuration between the standard multi-channel "frame transfer" mode and the single channel "spectra-kinetics" mode.

\section{CALIBRATION}

FIDA spectroscopy relies on absolutely calibrated spectra for a quantitative interpretation. The intensity calibration of the spectrometer is performed measuring the light produced by an integrating sphere (Labsphere, Model Unisource $\left.1200^{26}\right)$. The sphere produces a spectrum with a well known radiance. From the calibration the bar shadow is characterised and the conversion factors from digital counts to photon counts, [ph / ( $\left.\left.\mathrm{s} \mathrm{sr}^{2} \mathrm{~nm}\right)\right]$ is determined.

Correcting for camera effects such as the bias, smear and gain by electron multiplication is necessary before the calibration can be applied to the raw spectra. Several dark frames measured immediately after each discharge are used to correct for the EM-CCD charge bias. A non-illuminated channel is used to estimate the smearing in the multi-channel configuration. For spectra-kinetics a separate smear channel is not possible instead the effective exposure time is calculated considering the additional time pixels remain exposed to light as they are shifted. This additional exposure is equal to half the width of the ROI multiplied by the shift time. The multiplication of charge by use of the on chip EM register has been characterised. The gain is linear to about $1 \%$. To correct for the remaining part, detailed calibration is applied.

An absolute calibration of the spectrometer allows for a direct comparison with other spectrometers installed at ASDEX Upgrade. Figure 9 shows the calibration conversion factors of the new edge FIDA spectrometer compared to those of a core (COR) CXRS spectrometer ${ }^{25}$. Here the applied gain factors have been adjusted for and the difference in slit width between the two spectrometers taken into account. The COR spectrometer is an optimised $\mathrm{f} / 2.8$ spectrometer, using a similar but smaller ProEM 512×512 pixel EM-CCD camera. The new prototype spectrometer shows good photon throughput characteristics compared to the COR system at $650 \mathrm{~nm}$, especially for the central channels. 


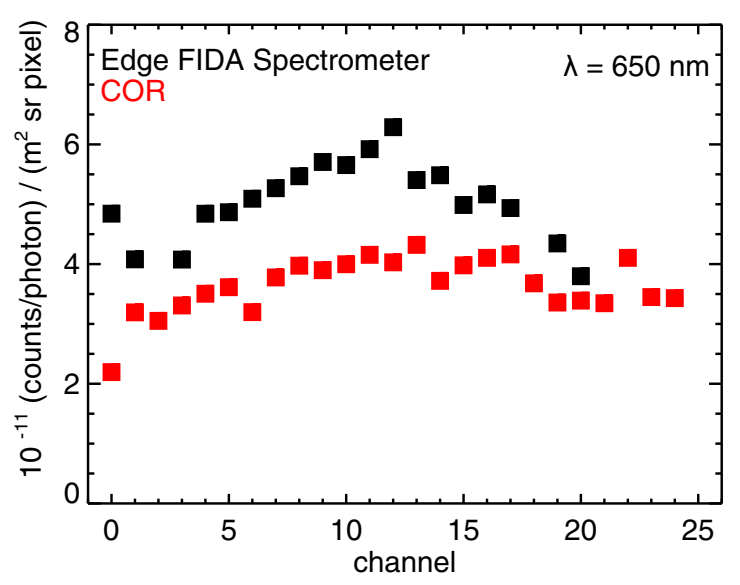

FIG. 9: Comparison of the spectrometer calibrations for the new edge FIDA spectrometer and the 'COR' CXRS spectrometer installed at ASDEX Upgrade.

Some uncertainty in the calculated spectral intensity can arise from transmission degradation of the optics due to coating of the in vessel objective lenses, or damage to the optical transmission fibres. In addition, uncertainty arises when switching fibres between the edge FIDA and CXRS system since the applied FC-connectors might not reproduce conditions by $100 \%$ when disconnected and connected again.

These sources of uncertainty are difficult to quantify. One routinely applied method is to compare experimental spectra to forward modelling of the neutral beam emission as suggested by Heidbrink et al. ${ }^{27}$. The expected emission of this contribution can be calculated using FIDASIM. Good agreement of the beam emission can be obtained for multiple channels simultaneously providing an indication of channels with unreliable fibre connections.

An accurate wavelength calibration is performed by measuring and fitting a forward model to the emission lines from a neon lamp, as are shown in figure 10. The wavelengths of $\mathrm{Ne}$ emission lines are well known and available from the NIST database $^{28}$. The forward model fits a synthetic spectrum constructed of Gaussian functions to the Ne spectrum for each channel. The free parameters of the grating equation ${ }^{29} \mathrm{ob}-$ tained from the fit are used to calculate the wavelength axis per channel. The spectral width of emission lines determines the spectral resolution of the spectrometer and is described as an instrument function. The instrument function is modelled here by a convolution between a box function and a Gaussian curve which well recovers the spectral shape, in part determined by the wide spectrometer entrance slit (box) and by the aberrations of the lenses and the grating (Gaussian). A comparison between the measured and fitted data is shown in the enlarged box in figure 10. The average instrument width of the spectrometer is $0.21 \mathrm{~nm}$.

The slight decrease in the intensity observed to the left of the emission lines in figure 10 appears to be a camera readout error associated with the sharply increased intensity. Fortunately this only affects a few pixels and is generally not ob- served in the wavelength ranges of interest of spectra measured during experiments.

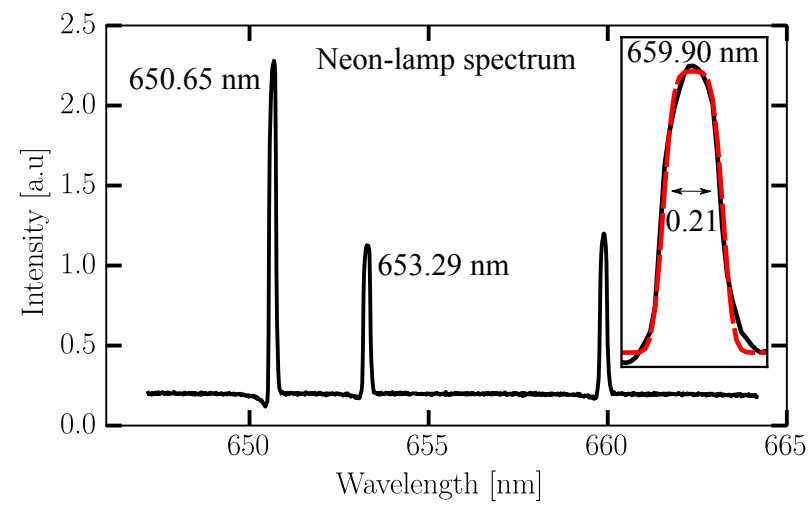

FIG. 10: Measured emission spectrum of a neon lamp used for wavelength calibration. A single neon emission line demonstrating the spectral resolution with the fitted instrument function plotted with dashed lines.

\section{EDGE FIDA MEASUREMENTS}

Initial edge FIDA measurements have been carried out with the spectrometer connected to 10 toroidal LOS of the edge CXRS system. For those multi-channel measurements every second LOS of the edge CXRS lines is used. In this manner edge ion temperature and velocity profiles can still be obtained, which is important for the analysis. Spectra measured during a low density H-mode discharge continuously heated by two off axis NBI beams (Q6 and Q7 in figure 1), with 50 ms long NBI phases of the active NBI beam (Q3) are shown in figure 11. The spectrum in red represents a passive spectrum (without NBI Q3), averaged over 4 sequential frames (10 ms). From left to right, the passive spectrum contains the $\mathrm{BV}$ and OV impurity lines followed by passive FIDA observable from about 650 to $655 \mathrm{~nm}$. The emission between about 655 and $658 \mathrm{~nm}$ originates from excited background neutrals whose density decays exponentially towards the plasma core. The emission exhibits intense and unshifted radiation from the cold edge neutrals and a broader feature from neutrals inside the last closed flux surface. The double peak observed for the unshifted D-alpha emission line appears to be a remnant of the bar shadow. To the right of these contributions are two CII impurity lines.

In addition the active+passive spectrum during operation of NBI Q3 is plotted in black, identifiable by the beam emission peaks (BES). Finally, a synthetic spectrum from FIDASIM is plotted against the measured spectra, with the sum of all contributions indicated by a blue dashed curve. The calculated emission indicates that the passive FIDA contribution (in magenta) is comparable in magnitude to the active contribution (in green).

The passive emission has been calculated with a neutral 
density profile modelled using the 1D neutral transport code $\mathrm{KN}_{1} \mathrm{D}^{30}$. KN1D calculates the neutral density profile in slab geometry given the plasma kinetic profiles and the neutral pressure near the wall, which is treated as a free parameter. The pressure measured by a manometer on the far outboard side, drawn in red in figure 1, is used as a reference value. The neutral density profile is scaled by multiplying the neutral pressure value with a factor that best reproduces the measured thermal CX contribution, as forward modelled by FIDASIM. In this manner the neutral density is constrained against the thermal CX emission and the passive FIDA contribution can be evaluated unambiguously. It should therefore be noted that the magenta curve represents both the passive FIDA and thermal CX contributions.

The lack of red shifted FIDA emission in the spectrum can be attributed to the predominantly co-passing fast-ion content generated by the off axis beams as illustrated in figure 4 .

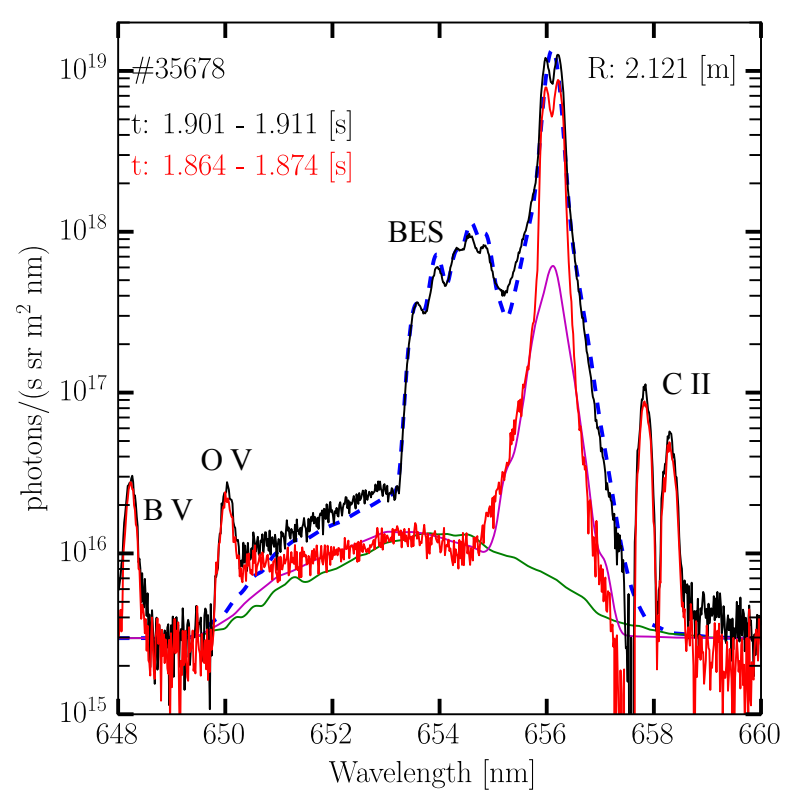

FIG. 11: Active (black) and passive (red) edge spectra measured during a discharge with $2.5 \mathrm{MW}$ of off-axis NBI heating. The active (green) and passive (magenta) FIDA contributions calculated by FIDASIM is over plotted with the sum of all contributions indicated by a blue dashed curve. The magenta line also includes the thermal CX contribution.

By integrating the spectra in wavelength for each LOS, radial profiles can be studied. Figure 12 shows a profile for the pure active signal (background subtraction has been applied) for wavelengths between 651 and $652.5 \mathrm{~nm}$ plotted in blue. The difference in LOS geometry of the standard and edge FIDA systems makes a direct comparison difficult as they probe slightly different regions of velocity space. The figure however illustrates the extended range acquired by the new edge system. The mismatch of the forward modelled profile with the measured profile outside the separatrix indicates that the assumed exponential decay in fast-ion density under estimates the true density.

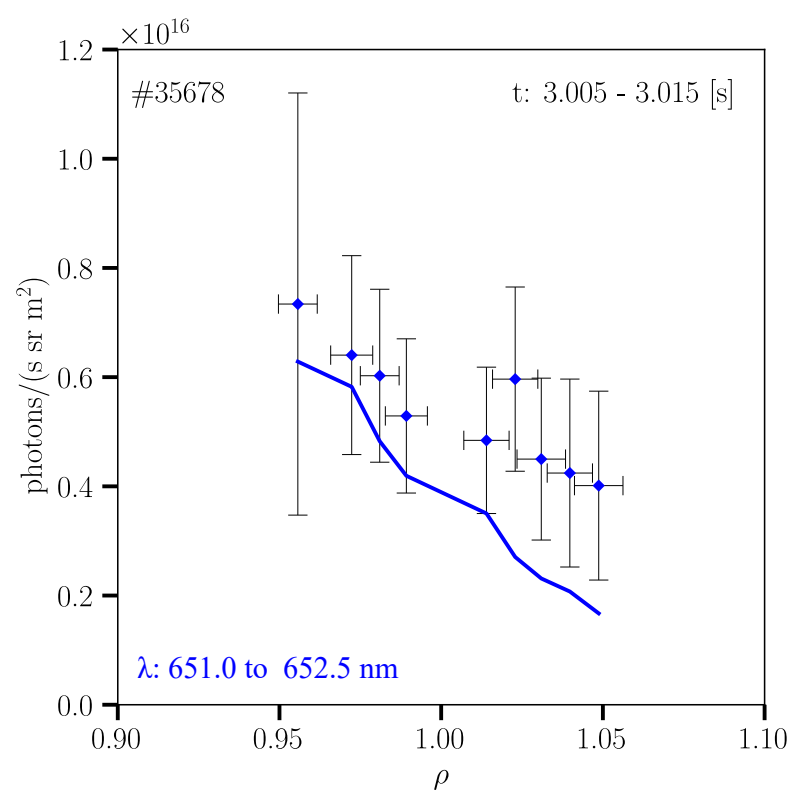

FIG. 12: Profile of active FIDA emission integrated over the indicated wavelength range of the spectra measured by the edge FIDA system. The solid line represents the profile obtained from FIDASIM calculated spectra.

\section{A. Fast edge FIDA measurements}

Edge FIDA measurements with exposure times of $200 \mu \mathrm{s}$ have been performed at ASDEX Upgrade making use of the spectra-kinetics readout mode. To increase the photon flux on the EM-CCD chip for a sufficient SNR the three inner most edge CXRS LOS are binned together. The tightly spaced LOS means the radial width probed is still less than $2 \mathrm{~cm}$ for active FIDA.

The presence of ELMs is observed to have a strong modulating effect on the FIDA emission. ELMs are know to cause fluctuations of FIDA emission ${ }^{31}$ and are therefore usually filtered out when analysing spectra. Figure 13 shows time traces of the passive FIDA emission, unshifted D-alpha emission and a divertor shunt current, for a discharge with a quasi regular ELM period of around $15 \mathrm{~ms}$. The shunt current serves as an ELM monitor where spikes in the current indicate ELM crashes as marked by the vertical green lines. The passive FIDA emission along the edge LOS strongly decreases during an ELM crash, and recovers after several milliseconds to preELM levels prior to the onset of the following ELM. Simultaneously the unshifted D-alpha emission strongly increases, inline with the experience of D-alpha emission as a proxy for ELM crashes.

Figure 14 shows the full, conditionally averaged spectra of the measurements presented in figure 13. The black spectrum is the average of frames -3.2 to $-3.0 \mathrm{~ms}$ relative to the ELM 

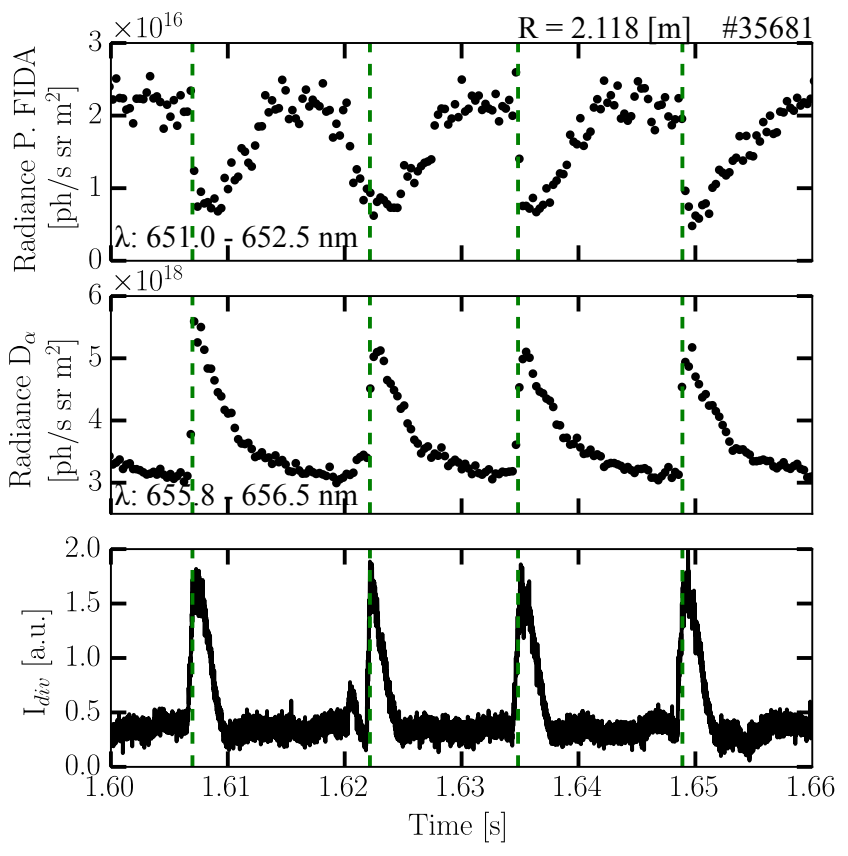

FIG. 13: Time trace of passive FIDA radiance (top), unshifted D-alpha radiance (middle) and divertor shunt current (bottom), during a time window of ELM crashes with a quasi regular frequency.

$\operatorname{crash}\left(\mathrm{t}_{E L M}=0 \mathrm{~s}\right)$, while the red spectrum is the averaged spectrum of frames measured 1.3 to $1.5 \mathrm{~ms}$ after the ELM crash. A clear reduction and flattening of the passive FIDA spectrum is observed, accompanied by increased unshifted D-alpha emission. This indicates a strong impact of ELMs on the edgefast-ion distribution function along the LOS which will be the topic of future publications. Here, we would like to emphasise that the new edge-FIDA capabilities reveal dynamics of the full D-alpha spectrum not resolvable on the standard FIDA diagnostic of ASDEX Upgrade.

\section{CONCLUSIONS AND OUTLOOK}

A new FIDA spectrometer has been installed at ASDEX Upgrade connected to viewing chords of the edge CXRS optical system. This extends the radial coverage of FIDA measurements at ASDEX Upgrade to beyond the separatrix. The spectrometer is able to measure the full deuterium Balmer alpha spectrum through the use of a blocking bar to create a narrow wedge filter. A choice between two readout modes of the EM-CCD camera allows for either multi-channel measurements with time resolution in the millisecond range, or single channel burst measurements down to the hundreds of microseconds. Multi-channel edge FIDA measurements have been carried out demonstrating good SNR and significant passive FIDA emission for discharges with off-axis NBI heating. Measurements with exposure times of $200 \mu$ s demonstrated reasonable SNR. This opens the possibility to investigate the effect of phenomena such as ELMs on the fast-ion distribu-

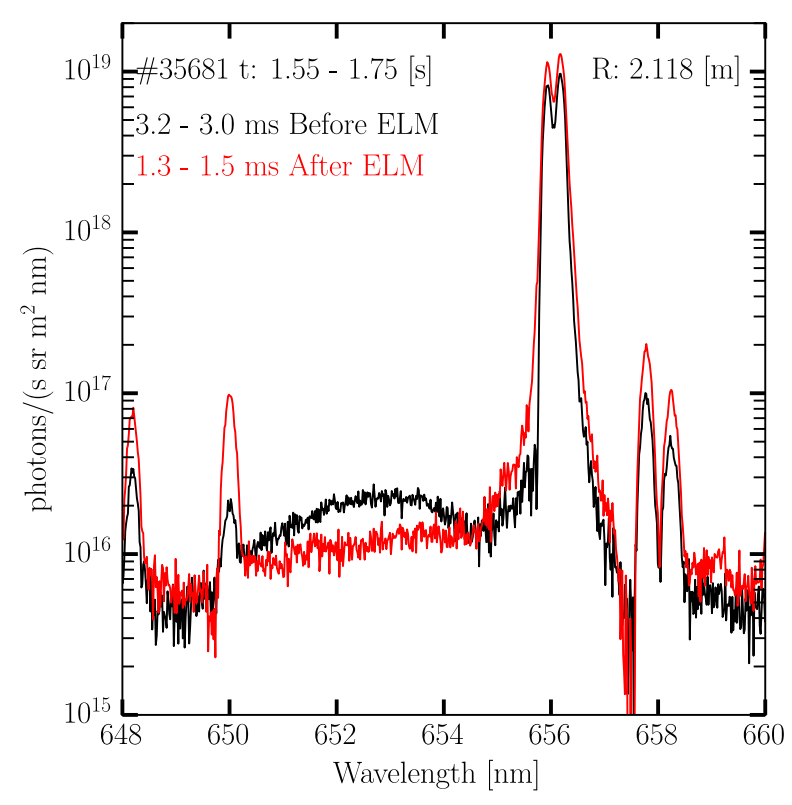

FIG. 14: Conditionally averaged pre- and post ELM spectra measured by the new edge FIDA spectrometer using the "spectra-kinetics" mode.

tion function. In addition future upgrades of the FIDA system with additional fast spectrometers would allow the analysis of profile-effects with $200 \mu$ s time resolution.

\section{ACKNOWLEDGEMENTS}

This work has been carried out within the framework of the EUROfusion Consortium and has received funding from the Euratom research and training programme 2014-2018 and 2019-2020 under grant agreement No 633053. The views and opinions expressed herein do not necessarily reflect those of the European Commission.

${ }^{1}$ A. Fasoli, C. Gormenzano, H. Berk, B. Breizman, S. Briguglio, D. Darrow, N. Gorelenkov, W. Heidbrink, A. Jaun, S. Konovalov, R. Nazikian, J.-M. Noterdaeme, S. Sharapov, K. Shinohara, D. Testa, K. Tobita, Y. Todo, G. Vlad, and F. Zonca, Nuclear Fusion 47, S264 (2007).

${ }^{2}$ D. Moseev, M. Salewski, M. Garcia-Muñoz, B. Geiger, and M. Nocente, Reviews of Modern Plasma Physics 2, 7 (2018).

${ }^{3}$ W. W. Heidbrink, K. H. Burrell, Y. Luo, N. A. Pablant, and E. Ruskov, Plasma Physics and Controlled Fusion 46, 1855 (2004).

${ }^{4}$ B. Geiger, R. Dux, R. M. McDermott, S. Potzel, M. Reich, F. Ryter, M. Weiland, D. Wünderlich, the ASDEX Upgrade Team, and M. Garcia-Munoz, Review of Scientific Instruments 84, 113502 (2013).

${ }^{5}$ M. Podestà, W. W. Heidbrink, R. E. Bell, and R. Feder, Review of Scientific Instruments 79, 10E521 (2008).

${ }^{6}$ M. Osakabe, S. Murakami, M. Yoshinuma, K. Ida, A. Whiteford, M. Goto, D. Kato, T. Kato, K. Nagaoka, T. Tokuzawa, Y. Takeiri, and O. Kaneko, Review of Scientific Instruments 79, 10E519 (2008).

${ }^{7}$ C. A. Michael, N. Conway, B. Crowley, O. Jones, W. W. Heidbrink, S. Pinches, E. Braeken, R. Akers, C. Challis, M. Turnyanskiy, A. Patel, D. Muir, R. Gaffka, and S. Bailey, Plasma Physics and Controlled Fusion 55, 095007 (2013). 
${ }^{8}$ C. M. Muscatello, W. W. Heidbrink, D. Taussig, and K. H. Burrell, Review of Scientific Instruments 81, 10D316 (2010).

${ }^{9}$ Y. M. Hou, C. R. Wu, J. Huang, W. W. Heidbrink, M. G. von Hellermann, Z. Xu, Z. Jin, J. F. Chang, Y. B. Zhu, W. Gao, Y. J. Chen, B. Lyu, R. J. Hu, P. F. Zhang, L. Zhang, W. Gao, Z. W. Wu, Y. Yu, and M. Y. Ye, Review of Scientific Instruments 87, 11E552 (2016).

${ }^{10}$ B. Geiger, A. N. Karpushov, B. P. Duval, C. Marini, O. Sauter, Y. Andrebe, D. Testa, M. Marascheck, M. Salewski, P. A. Schneider, the TCV Team, and the EUROfusion MST1 Team, Plasma Physics and Controlled Fusion 59, 115002 (2017).

${ }^{11}$ W. W. Heidbrink, D. Liu, Y. Luo, E. Ruskov, and B. Geiger, Communications in Computational Physics 10, 716-741 (2011).

${ }^{12}$ M. Salewski, B. Geiger, S. Nielsen, H. Bindslev, M. García-Muñoz, W. Heidbrink, S. Korsholm, F. Leipold, F. Meo, P. Michelsen, D. Moseev, M. Stejner, G. Tardini, and the ASDEX Upgrade Team, Nuclear Fusion 52, 103008 (2012).

${ }^{13}$ M. V. Zeeland, N. Ferraro, B. Grierson, W. Heidbrink, G. Kramer, C. Lasnier, D. Pace, S. Allen, X. Chen, T. Evans, M. García-Muñoz, J. Hanson, M. Lanctot, L. Lao, W. Meyer, R. Moyer, R. Nazikian, D. Orlov, C. PazSoldan, and A. Wingen, Nuclear Fusion 55, 073028 (2015).

${ }^{14}$ O. M. Jones, M. Cecconello, K. G. McClements, I. Klimek, R. J. Akers, W. U. Boeglin, D. L. Keeling, A. J. Meakins, R. V. Perez, S. E. Sharapov, M. Turnyanskiy, and the MAST team, Plasma Physics and Controlled Fusion 57, 125009 (2015).

${ }^{15}$ N. G. Bolte, W. W. Heidbrink, D. Pace, M. V. Zeeland, and X. Chen, Nuclear Fusion 56, 112023 (2016).

${ }^{16}$ G. Z. Hao, W. W. Heidbrink, D. Liu, M. Podesta, L. Stagner, R. E. Bell, A. Bortolon, and F. Scotti, Plasma Physics and Controlled Fusion 60, 025026 (2018).

${ }^{17}$ E. Viezzer, T. Pütterich, R. Dux, R. M. McDermott, and the ASDEX Upgrade Team, Review of Scientific Instruments 83, 103501 (2012).

${ }^{18}$ M. Cavedon, T. Pütterich, E. Viezzer, R. Dux, B. Geiger, R. M. McDermott, H. Meyer, U. Stroth, and the ASDEX Upgrade Team, Review of Scientific Instruments 88, 043103 (2017).
${ }^{19} \mathrm{~B}$. Geiger, Fast-ion transport studies using FIDA spectroscopy at the ASDEX Upgrade tokamak, Ph.D. thesis, Ludwig Maximilian University of Munich (2013).

${ }^{20}$ A. Pankin, D. McCune, R. Andre, G. Bateman, and A. Kritz, Computer Physics Communications 159, 157 (2004).

${ }^{21}$ See www. newport.com/p/RVS80CC for information on the rotation stage used.

${ }^{22}$ See http://www.princetoninstruments.com for Princeton Instruments.

${ }^{23}$ Y. Luo, W. W. Heidbrink, K. H. Burrell, D. H. Kaplan, and P. Gohil, Review of Scientific Instruments 78, 033505 (2007).

${ }^{24}$ M. Weiland, B. Geiger, A. S. Jacobsen, M. Reich, M. Salewski, T. Odstrčil, and the ASDEX Upgrade Team, Plasma Physics and Controlled Fusion 58, 025012 (2016).

${ }^{25}$ R. M. McDermott, A. Lebschy, B. Geiger, C. Bruhn, M. Cavedon, M. Dunne, R. Dux, R. Fischer, A. Kappatou, T. Pütterich, E. Viezzer, and the ASDEX Upgrade Team, Review of Scientific Instruments 88, 073508 (2017).

${ }^{26}$ See http://www.labsphere.com for information on the integrating sphere which has been used for the intensity calibration.

${ }^{27}$ W. W. Heidbrink, A. Bortolon, C. M. Muscatello, E. Ruskov, B. A. Grierson, and M. Podestá, Review of Scientific Instruments 83, 10D903 (2012).

${ }^{28}$ A. Kramida, Yu. Ralchenko, J. Reader, and NIST ASD Team, NIST Atomic Spectra Database (ver. 5.6.1), [Online]. Available: https://physics.nist.gov/asd [2019, May 23]. National Institute of Standards and Technology, Gaithersburg, MD. (2018).

${ }^{29}$ R. E. Bell, Review of Scientific Instruments 75, 4158 (2004).

${ }^{30}$ B. LaBombard, "KN1D: A 1-D space kinetic, 2-D velocity, transport algorithm for atomic molecular hydrogen in an ionizing plasma," Research Report PSFC-RR-01-3 (Massachusetts Institute of Technology, Plasma Science and Fusion Center, 175 Albany St., Cambridge, MA 02139 USA, 2001).

${ }^{31}$ B. Geiger, M. Garcia-Munoz, W. W. Heidbrink, R. M. McDermott, G. Tardini, R. Dux, R. Fischer, V. Igochine, and the ASDEX Upgrade Team, Plasma Physics and Controlled Fusion 53, 065010 (2011). 